\title{
Behavioral investigation of the influence of social categorization on empathy for pain: a minimal group paradigm study
}

\author{
Benoît Montalan ${ }^{1,2,3}$, Thierry Lelard ${ }^{1,3}$, Olivier Godefroy ${ }^{1,3,4}$ and Harold Mouras ${ }^{1,3}$ * \\ 1 Laboratoire de Neurosciences Fonctionnelles et Pathologies, UFR de Médecine, Université de Picardie Jules Verne, Amiens, France \\ ${ }^{2}$ Laboratoire de Psychologie et Neurosciences: Intégration Cognitive du Neurone à la Société, Université de Rouen, Mont-Saint-Aignan, France \\ ${ }^{3}$ Structure Fédérative de Recherche en Santé CAP-Santé, Université de Picardie Jules Verne, Amiens and Université de Reims-Champagne-Ardennes, Reims, France \\ ${ }^{4}$ Service de Neurologie, Center Hospitalier Universitaire d'Amiens, Amiens, France
}

\section{Edited by:}

Narayanan Srinivasan, University of Allahabad, India

\section{Reviewed by:}

David Vaughn Becker, Arizona State University, USA

Mark Levine, Exeter University, UK

*Correspondence:

Harold Mouras, Laboratoire de

Neurosciences Fonctionnelles et

Pathologies, Site d'Amiens, UFR de

Médecine, 3 Rue des Louvels, 80000

Amiens, France.

e-mail: harold.mouras@u-picardie.fr
Research on empathy for pain has provided evidence of an empathic bias toward racial ingroup members. In this study, we used for the first time the "minimal group paradigm" in which participants were assigned to artificial groups and required to perform pain judgments of pictures of hands and feet in painful or non-painful situations from self, ingroup, and outgroup perspectives. Findings showed that the mere categorization of people into two distinct arbitrary social groups appears to be sufficient to elicit an ingroup bias in empathy for pain.

Keywords: empathy for pain, ingroup bias, social categorization, minimal group paradigm

\section{INTRODUCTION}

Empathy is thought to play a critical role in social interactions in motivating prosocial behavior (e.g., Dovidio et al., 1990). This human ability is a psychological construct regulated by both cognitive and affective components (e.g., Decety and Jackson, 2004; Shamay-Tsoory et al., 2009). The affective component involves sharing the emotional experiences of others, while the cognitive component involves thinking about and understanding the mental states of others.

Research on the psychological and neural mechanisms of empathy has substantially grown this past decade, focusing more specifically on how we share the pain of others, one of the most basic and universal human experiences. In this regard, it has been recently demonstrated that response to other's pain depends on the social relationships between the observer and the individuals experiencing the outcome. For example, affective links (e.g., Singer et al, 2006), perceived similarities (e.g., Perry et al., 2010), and social memberships (e.g., Xu et al., 2009) are likely to modulate the level of empathy experienced by the observer toward agent's pain. More precisely, number of studies on empathy for pain in intergroup contexts has provided evidence of an empathic bias toward racial ingroup members (Xu et al., 2009; Avenanti et al., 2010; see Chiao and Mathur, 2010, for a review).

In the field of social psychology, it is well-known that people are remarkably adept at dividing up the social world into us versus them, and that this propensity has important affective, cognitive, and behavioral consequences such as prejudice, stereotype, and discrimination. These various implications are particularly induced when categorical information such as race, gender, or age, provide visually salient cues to group membership (Fiske and Neuberg, 1990). However, studies based on the Minimal Group
Paradigm (MGP; Tajfel et al., 1971) have demonstrated that the mere categorization of individuals into two social groups on the basis of arbitrary criteria, such as whether they tend to overestimate or underestimate the number of dots on a screen (Diehl, 1990), is sufficient to produce similar consequences as compared to natural groups. Now, no studies used the MGP, in research on empathy for pain. Thus, the present study examined whether the mere categorization of people into distinct arbitrary social groups is sufficient to elicit an ingroup bias in empathy for pain. To this end, participants were shown pictures of people in painful or nonpainful situations and were instructed to imagine themselves or imagine members of two minimal groups (ingroup vs. outgroup) in the same situations. Participants then had to rate the level of perceived pain according to the different perspectives (e.g., Jackson et al., 2006).

\section{MATERIALS AND METHODS PARTICIPANTS}

Thirty-six native French speakers were included (eight males; mean age $=21.5 \pm 2.2$ ). None had prior or current treatment for any psychiatric disorder or neurological condition (Godefroy et al., 2010). The study conformed to the IASP's guidelines and was approved by the local ethics committee (CPP Nord Ouest 2, Amiens, France). All participants provided informed written consent.

\section{PROCEDURE}

Upon arrival in the lab, participants provided informed consent and were asked to fill out the Interpersonal Reactivity Index (IRI; Davis, 1983; French version: Guttman and Laporte, 2002). The IRI is a multidimensional measure composed of 28 self-report items 
using a 5-point Likert scale and designed to evaluate different dimensions of empathy. The subscales of the IRI consist of four subscales of seven items. The Fantasy $(F)$ subscale assesses the tendency to imaginatively transpose oneself into fictional situations (e.g., I really get involved with the feelings of the characters in a novel). The Perspective Taking (PT) subscale assesses the tendency to spontaneously adopt the psychological point of view of others (e.g., I try to look at everybody's side of a disagreement before I make a decision). The Empathic Concern (EC) subscale assesses the tendency to experience feelings of sympathy and concern for others in distress (e.g., I often have tender, concerned feelings for people less fortunate than me). The Personal Distress (PD) subscale assesses the tendency to experience distress and discomfort in response to distress in others (e.g., In emergency situations, I feel apprehensive, and ill-at-ease). In the present study, and as in previous ones (e.g., Rankin et al., 2006), we chose to focus on two IRI subscales only, which measure the cognitive (PT subscale) and affective (EC subscale) components of empathy.

After filling out the IRI, participants were informed that the session was part of a larger project investigating the relationship between cognition and emotion. They were comfortably seated at a viewing distance of $70 \mathrm{~cm}$ from a computer monitor and instructed to perform a cognitive task in order to determine their cognitive profile. A dot-estimation task was used to divide subjects into two fictive groups: 18 participants were categorized as underestimators, i.e., those who allegedly had underestimated the number of dots in a series of stimulus displays, while 18 others were categorized as overestimators, i.e., those who allegedly had overestimated the number of dots in a series of stimulus displays. Finally, the experimenter gave additional information to the participants about the supposed differences between the two groups in order to reinforce their feeling of membership (see also Ashburn-Nardo et al., 2001; Pinter and Greenwald, 2011) and, more specifically, to avoid potential effects of asymmetry in terms of status or power (e.g., Sachdev and Bourhis, 1987; e.g., "Previous research has shown that people who overestimate the number of dots tend to process perceptual information in a bottom-up fashion. That is, you tend to examine the finer details of new stimuli, and then form an overall impression. In contrast, people who underestimate the number of dots tend to process perceptual information in a top-down fashion. That is, they tend to form an overall impression, and then examine the finer details of new stimuli. However, none of these two modes of processing is better than the other one.").

Just after the minimal categorization procedure, participants performed an emotion task, i.e., a pain judgment task in which they were shown 36 static visual stimuli, consisting of 18 color pictures showing hands and feet in painful situations and 18 color pictures of hands and feet in non-painful situations (Jackson et al., 2005). Adobe Photoshop ${ }^{\circledR}$ (Adobe Systems, Inc.) was used to resize the images to approximately $500 \times 375$ pixels (screen resolution: $1024 \times 768$ pixels). The stimuli were presented in three blocks (themselves, members of the ingroup, and member of the outgroup) of 36 trials each, for a total of 108 trials. At the beginning of each block, the participants were instructed to imagine themselves, a member of the ingroup, or a member of the outgroup in the displayed situations, and to rate the level of perceived pain according to the different perspectives. The order of the blocks was counterbalanced on a between-subjects basis. The trial sequence started with a fixation cross for $500 \mathrm{~ms}$. Then the stimulus was presented until participants responded. Immediately after the onset of the stimulus, subjects were instructed to deliver their ratings by pressing with the right hand one of nine computer keys (with scores ranging from $0=$ no pain to $8=$ very severe pain). After responses, an inter-stimulus interval (ISI) of $1000 \mathrm{~ms}$ was added.

Following the pain judgment task, subjects completed a 20-item adjective rating scale, including 10 positive items and 10 negative items (Montalan et al., 2011), to measure ingroup bias. The negative stimuli had a mean valence rating of $-3.04 \pm 1.47$, while the positive stimuli had a mean valence rating of $3.85 \pm 0.93$. Participants rated how descriptive each item was for each target group. Responses range from $0=$ does not describe to $8=$ describes completely. The negative adjectives of the scale were reverse scored. Thus, an evaluative score (ES) used to measure ingroup bias was calculated by subtracting for each participant the outgroup evaluation score from the ingroup evaluation score (outgroup bias: $\mathrm{ES}<0$, no bias: $\mathrm{ES}=0$, and ingroup bias: $\mathrm{ES}>0$ ).

\section{STATISTICAL ANALYSES}

For the pain judgment task, pain ratings and response times were submitted to two repeated-measure analyses of variance (ANOVA) with Stimulus (Pain vs. No-pain) and Perspective (Self vs. Ingroup vs. Outgroup) as within-subject factors. For the ingroup bias measure, difference of evaluation scores from zero was evaluated with a one-sample $t$-test. Finally, Pearson's correlation coefficients between the subjects' performance in the pain judgment task, the evaluation scores and the IRI scores (PT and EC scores) were also calculated. For all statistical analyses, the $\alpha$ was fixed at $5 \%$.

\section{RESULTS \\ PAIN JUDGMENT TASK \\ Pain ratings}

The two-factor ANOVA revealed a significant effect for Stimulus, due to higher ratings for pain stimuli $(M=5.32 \pm 1.45)$ than for no-pain stimuli $(M=0.49 \pm 0.74), F(1,35)=634.77, p<0.001$. However, a significant interaction effect between Stimulus and Perspective (Figure 1) was also found, $F(2,70)=3.29, p<0.05$. Firstly, and according to our hypothesis, the difference in ratings of the pain and no-pain stimuli was calculated for each of the three types of perspectives. This difference was significantly higher for the ingroup perspective $(M=5.11 \pm 1.25)$ as compared to the outgroup perspective $(M=4.55 \pm 1.59), F(1,35)=5.29, p=0.05$. Secondly, we also compared the three types of perspectives with each other for the pain and no-pain stimuli respectively. For the no-pain stimuli, no significant differences were found. In contrast, pain ratings were significantly higher in the ingroup perspective $(M=5.54 \pm 1.31)$ than in the self-perspective $(M=5.16 \pm 1.33)$ for the pain stimuli, $F(1,35)=6.04, p<0.05$, no significant differences being observed with the two others comparisons (self vs. ingroup and ingroup vs. outgroup).

\section{RESPONSE TIMES}

The statistical analysis of response times (RTs) revealed a significant main effect for Stimulus, due to longer RTs for pain stimuli 


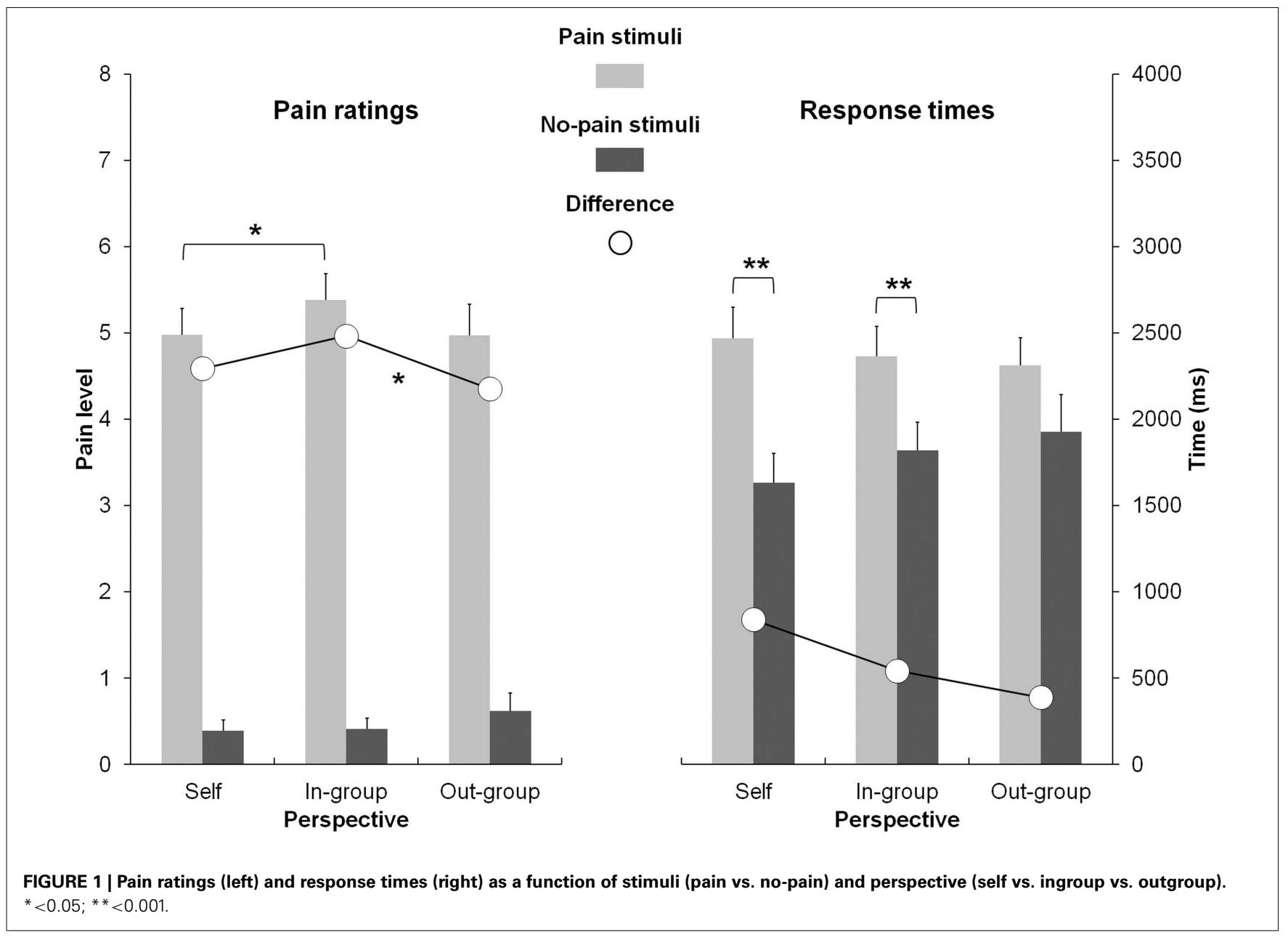

$(M=2370 \pm 847)$ than for no-pain stimuli $(M=1787 \pm 961)$, $F(1,35)=31.11, p<0.001$. However, the interaction between Stimulus and Perspective (Figure 1) was significant, $F(1$, $35)=5.51, p<0.01$. Planned comparisons disclosed that the responses for pain stimuli were significantly longer than those for no-pain stimuli in the self-perspective, $F(1,35)=53.80, p<0.001$, and in the ingroup perspective, $F(1,35)=18.09, p<0.001$.

\section{EVALUATIVE INGROUP BIAS}

The mean evaluation score was positive $(M=1.19 \pm 1.61)$ and a one-sample $t$-test revealed that it significantly differs from zero, $t(1,35)=4.45, p<0.001$. As shown in Table 1 , no correlation coefficients between the subjects' evaluation scores and the performance in the pain judgment task reached significance.

\section{EMPATHY MEASURE}

The descriptive scores of the IRI and the correlation results are reported in Table 1. As shown, a significant negative relation between the EC scores and the ratings of no-pain stimuli in the ingroup perspective was observed, $r=-0.39$; $p<0.05$. Moreover, a positive relation between the EC scores and the RTs for the Pain/No-pain difference in the ingroup perspective reached also significance, $r=0.41 ; p<0.05$.

\section{DISCUSSION}

In the present study, we replicated previous findings showing that the mere assignment of individuals to arbitrary groups (i.e., the MGP) elicits evaluative preferences for ingroup relative to outgroup members (Brewer, 1979). More interestingly, we found that the mere act of categorizing people in two distinct social groups is also sufficient to elicit an ingroup bias in empathy for pain ${ }^{1}$. Indeed, participants rated pain stimuli as more painful when they had to adopt the perspective of an ingroup's member as compared to their own perspective, while the outgroup perspective did not induce different responses to painful pictures as compared to the self-perspective. Moreover, the ratings differences

\footnotetext{
${ }^{1}$ One might argue that by highlighting the distinction between the two groups - or introducing information relevant to power or status relations (see Method section), they were not minimal in the accepted sense (Tajfel et al., 1971). Thus, the effects we found would be a function of interpretations of the content we provided - whereas additional information was devoid of any positive or negative evaluation- rather than the mere categorization. It is worth to notice that some authors have used a similar procedure (Ashburn-Nardo et al., 2001; Pinter and Greenwald, 2011), but none of them pointed this aspect. Moreover, given that our method does not allow us to clarify this issue, further investigations should be conducted in order to make sure that the mere act of categorizing people in two distinct social groups is sufficient to elicit an ingroup bias in empathy for pain, for example by comparing performances in two paradigms with and without these instructions.
} 
Table 1 | Cognitive (the Perspective Taking subscale) and affective (the Empathic Concern subscale) subscales of the Interpersonal Reactivity Index (IRI; Davis, 1983; French version: Guttman and Laporte, 2002), evaluation scores, and correlation results.

Interpersonal reactivity index

Evaluation score

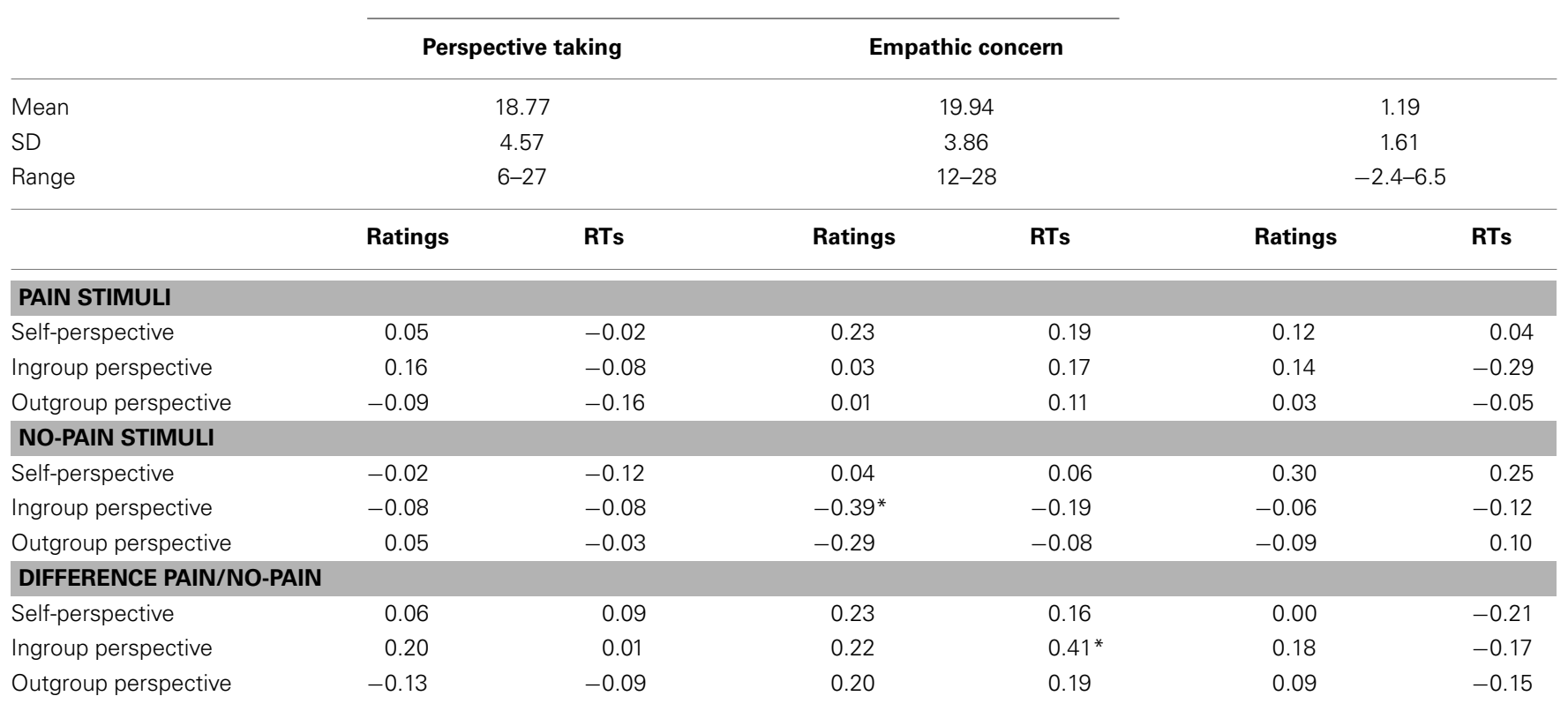

${ }^{*} p<0.05$.

between the painful and non-painful pictures were more important in the ingroup perspective than in the outgroup perspective. Taken together, these observations are consistent with a more general empathic bias toward ingroup members previously shown in the field of intergroup relations (e.g., Brown et al., 2006; Tarrant et al., 2009). For example, some authors found that participants reported stronger empathy when a student in distress belonged to an ingroup compared to an outgroup university (Tarrant et al., 2009). In a similar way, Brown and collaborators (2006) provided evidence for an empathic ingroup bias, participants showing exaggerated affective responses to positive and negative pictures depicting ingroup members.

Contrary to previous studies (e.g., Jackson et al., 2006; Li and Han, 2009), we did not find that taking self-perspective induced faster reactions to perceived pain. In contrast, we found participants to respond more quickly to non-painful than painful stimuli when taking self or ingroup perspective, but not when taking outgroup perspective. Such a discrepancy may be due to methodological differences. In previous researches, response times were collected based on two-alternative forced-choice tasks (i.e., subjects had to judge painful vs. non-painful pictures on each trial) so that the subjects can assign a simple, repeatable, and differentiated motor response to each decision. In contrast, in the present case, reaction times were measured as participants had to rate the level of perceived pain according to the different perspectives. Thus, slower reactions to perceived pain in the self and in groupperspectives could be due to the more care took in accurately assigning a number to pain felt, or alternatively, perhaps an additional stage of processing. Regardless of eventual explanations, it is worth to notice that the same pattern was found when participants had to adopt their own perspective or the one of an ingroup's member, suggesting the implication of the same mechanisms in these two conditions.

It is well documented in social psychological literature that when two individuals become close, the other is integrated into the self-concept (e.g., Aron et al., 1991). In this vein, Smith and Henry (1996) demonstrated that self-other overlap in mental representations was more important for individuals defined as ingroup members as compared to outgroup members. Thus, an evaluative ingroup bias would be based on the individuals' proclivity to extend their positive self-representations to encompass their groups (e.g., Cadinu and Rothbart, 1996; Otten and Epstude, 2006). In a similar way, to the extent that similarity between oneself and another may have an important impact on the level of empathy experienced toward another (e.g., Batson et al., 1995; Perry et al., 2010), this overlapping mental representations of the self and the ingroup may also explain the empathic ingroup bias. As compared to previous studies showing that failures of empathy toward outgroup members potentially make them likely targets for prejudice and discrimination (e.g., Gutsell and Inzlicht, 2010), we did not find, however, any relationship between the evaluative and empathic ingroup biases.

Our results also revealed that the cognitive $(P T)$ subscale of the (IRI; Davis, 1983; French version: Guttman and Laporte, 2002) did not predict the participants' performance in the pain judgment task. This suggests that ingroup bias in empathy for pain would not be based on the individuals' abilities to adopt the perspective of the other. In contrast, the pain judgments in the ingroup perspective were correlated to the EC subscale of the IRI. This confirms the critical role of the affective component, i.e., the affect produced in 
response to someone else's emotional state, in empathic ingroup bias for pain, as suggested by antecedent neural investigations (e.g., Xu et al., 2009).

In conclusion, the present study provided new evidence that an observer feels more empathy for someone in pain when that person is in the same social group. But more importantly, we showed for the first time that the mere categorization of individuals on the basis of minimal criteria is sufficient to elicit such an ingroup bias in empathy for pain. Further investigations should be conducted

\section{REFERENCES}

Aron, A., Aron, E. N., Tudor, M., and Nelson, G. (1991). Close relationships as including other in the self. J. Pers. Soc. Psychol. 60, 241-253.

Ashburn-Nardo, L., Voils, C. I., and Monteith, M. J. (2001). Implicit associations as the seeds of inter group bias: how easily do they take root? J. Pers. Soc. Psychol. 81, 789-799.

Avenanti, A., Sirigu, A., and Aglioti, S. M. (2010). Racial bias reduces empathic sensorimotor resonance with otherrace pain. Curr. Biol. 20, 1018-1022.

Batson, C. D., Turk, C. L., Shaw, L. L., and Klein, T. R. (1995). Information function of empathic emotion: learning that we value the other's welfare. J. Pers. Soc. Psychol. 68, 300-313.

Brewer, M. B. (1979). In-group bias in the minimal intergroup situation: a ecognitive motivational analysis. Psychol. Bull. 86, 307-324.

Brown, L. M., Bradley, M. M., and Lang, P. J. (2006). Affective reactions to pictures of ingroup and outgroup members. Biol. Psychol. 71, 303-311.

Cadinu, M. R., and Rothbart, M. (1996). Self-anchoring and differentiation processes in the minimal group paradigm. J. Pers. Soc. Psychol. 70, 661677.

Chiao, J. Y., and Mathur, V. A. (2010). Intergroup empathy: how does race affect empathic neural response? Curr. Biol. 20, 478-480.

Davis, M. H. (1983). Measuring individual-differences in empathy evidence for a multidimensional approach. J. Pers. Soc. Psychol. 44, 113-126.

Decety, J., and Jackson, P. L. (2004). The functional architecture of human empathy. Behav. Cogn. Neurosci. Rev. 3, 71-100.

Diehl, M. (1990). “The minimal group paradigm: theoretical explanations and empirical findings," in European Review of Social Psychology, Vol. 1, eds W. Stroebe and M. Hewstone (Chichester: Wiley), 263-292.

Dovidio, J. F., Allen, J. L., and Schroeder, D. A. (1990). The specificity of empathy-induced helping: evidence for altruistic motivation. J. Pers. Soc. Psychol. 59, 249-260.

Fiske, S. T., and Neuberg, S. L. (1990). "A continuum of impression formation, from category-based to individuating processes: influences of information and motivation on attention and interpretation," in Advances in Experimental Social Psychology, Vol. 23, ed. M. P. Zanna (San Diego, CA: Academic Press), 1-74.

Godefroy, O., Azouvi, P., Robert, P., Roussel, M., LeGall, D., MeUlemans, T., et al. (2010). Dysexecutive syndrome: diagnostic criteria and validation study. Ann. Neurol. 68, 855-864

Gutsell, J. N., and Inzlicht, M. (2010). Empathy constrained: prejudice predicts reduced mental simulation of actions during observation of outgroups. J. Exp. Soc. Psychol. 46, 841-845.

Guttman, H., and Laporte, L. (2002). Alexithymia, empathy, and psychological symptoms in a family context. Compr. Psychiatry 43, 448-455.

Jackson, P. L., Brunet, E., Meltzoff, A. N., and Decety, J. (2006). Empathy examined through the neural mechanisms involved in imagining how I feel versus how you feel pain. Neuropsychologia 44, 752-761.

Jackson, P. L., Meltzoff, A. N., and Decety, J. (2005). How do we perceive the pain of others? A window into the neural processes involved in empathy. Neuroimage 24, 771-779.

Li, W., and Han, S. (2009). Perspective taking modulates event related potentials to perceived pain. Neurosci. Lett. 469, 328-332.

to explore the neural basis of this minimal ingroup bias in line with previous neural researches on empathy for pain in intergroup contexts (Xu et al., 2009; Avenanti et al., 2010; Gutsell and Inzlicht, 2010; for a review, see Chiao and Mathur, 2010).

\section{ACKNOWLEDGMENTS}

Benoît Montalan and Thierry Lelard are funded by the regional council of Picardie and the FEDER. The authors thank Jean Decety for the use of his database of stimuli.

Montalan, B., Boitout, A., Veujoz, M., Leleu, A., Germain, R., Personnaz, B., et al. (2011). Social identity-based motivation modulates attention bias toward negative information: an event-related brain potential study. Socioaffective Neurosci. Psychol. 1, 5892.

Otten, S., and Epstude, K. (2006). Overlapping mental representations of self, ingroup and outgroup: unraveling self-stereotyping and selfanchoring. Pers. Soc. Psychol. Bull. 32, 957-969.

Perry, A., Bentin, S., Ben-Ami Bartal, I., Lamm, C., and Decety, J. (2010). Feeling the pain of those who are different from us - modulation of EEG in the mu/alpha range. Cogn. Affect. Behav. Neurosci. 10, 493-504.

Pinter, B., and Greenwald, A. G. (2011). A comparison of minimal group induction procedures. Group Process. Interpers. Relations 14, 81-98.

Rankin, K. P., Gorno-Tempini, M. L. Allison, S. C., Stanley, C. M., Glenn, S., Weiner, M. W., et al. (2006). Structural anatomy of empathy in neurodegenerative disease. Brain 129, 2945-2956.

Sachdev, I., and Bourhis, R. Y. (1987) Status differentials and intergroup behaviour. Eur. J. Soc. Psychol. 17, 277-293.

Shamay-Tsoory, S. G., Aharon-Peretz, J., and Perry, D. (2009). Two systems for empathy: a double dissociation between emotional and cognitive empathy in inferior frontal gyrus versus ventromedial prefrontal lesions. Brain 132, 617-627.

Singer, T., Seymour, B., O’Doherty, J. P., Stephan, K. E., Dolan, R. J., and Frith, C. D. (2006). Empathic neural responses are modulated by the perceived fairness of others. Nature 439, 466-469.
Smith, E. R., and Henry, S. (1996). An in-group becomes part of the self: response time evidence. Pers. Soc. Psychol. Bull. 22, 635-642.

Tajfel, H., Billig, M., Bundy, R., and Flament, C. (1971). Social categorization and intergroup behaviour. Eur. J. Soc. Psychol. 1, 149-178.

Tarrant, M., Dazeley, S., and Cottom, T. (2009). Social categorization and empathy for outgroup members. Br. J. Soc. Psychol. 48, 427-446.

Xu, X., Zuo, X., Wang, X., and Han, S. (2009). Do you feel my pain? Racial group membership modulates empathic neural responses. $J$. Neurosci. 29, 8525-8529.

Conflict of Interest Statement: The authors declare that the research was conducted in the absence of any commercial or financial relationships that could be construed as a potential conflict of interest.

Received: 30 May 2012; accepted: 18 September 2012; published online: 17 October 2012.

Citation: Montalan B, Lelard T, Godefroy $O$ and Mouras H (2012) Behavioral investigation of the influence of social categorization on empathy for pain: a minimal group paradigm study. Front. Psychology 3:389. doi: 10.3389/fpsyg.2012.00389

This article was submitted to Frontiers in Cognitive Science, a specialty of Frontiers in Psychology.

Copyright (c) 2012 Montalan, Lelard, Godefroy and Mouras. This is an openaccess article distributed under the terms of the Creative Commons Attribution License, which permits use, distribution and reproduction in other forums, provided the original authors and source are credited and subject to any copyright notices concerning any third-party graphics etc. 